\title{
The relationship between central lymph node metastasis and the distance from tumor to thyroid capsule in papillary thyroid microcarcinoma without capsule invasion
}

\author{
Mengting Zhu ${ }^{1}$, Weihui Zheng ${ }^{2}$, Yangfeng Xiang ${ }^{2}$, Jialei Gu ${ }^{2}$, Kejing Wang ${ }^{2}$, Jinbiao Shang ${ }^{2}$ \\ ${ }^{1}$ The Second Clinical Medical College, Zhejiang Chinese Medical University, Hangzhou, China; ${ }^{2}$ Department of Head and Neck Surgery, Cancer \\ Hospital of the University of Chinese Academy of Sciences (Zhejiang Cancer Hospital); Institute of Cancer and Basic Medicine (IBMC), Chinese \\ Academy of Sciences; Key Laboratory of Head \& Neck Cancer Translational Research of Zhejiang Province, Hangzhou, China \\ Contributions: (I) Conception and design: J Shang; (II) Administrative support: J Shang; (III) Provision of study materials or patients: W Zheng; (IV) \\ Collection and assembly of data: M Zhu; (V) Data analysis and interpretation: M Zhu; (VI) Manuscript writing: All authors; (VII) Final approval of \\ manuscript: All authors. \\ Correspondence to: Jinbiao Shang. Department of Head and Neck Surgery, Cancer Hospital of the University of Chinese Academy of Sciences \\ (Zhejiang Cancer Hospital); Institute of Cancer and Basic Medicine(IBMC), Chinese Academy of Sciences; Key Laboratory of Head \& Neck Cancer \\ Translational Research of Zhejiang Province, Hangzhou, China. Email: shangjb@zjcc.org.cn.
}

Background: This study aims to explore the predictive factors of central lymph node metastasis (CLNM) in patients with papillary thyroid microcarcinoma (PTMC) without capsule invasion.

Methods: From January 2016 to October 2018, 1,622 patients with PTMC, who underwent surgical treatment at Zhejiang Cancer Hospital, were enrolled in the present study. A model of multivariate logistic regression was developed to find the variables that were independently associated with CLNM. The results were presented in the odds ratio (OR) with a 95\% confidence interval (CI). The nomogram for predicting CLNM was developed based on the results of the multivariate logistic regression analysis. The distance (distance $>0$ ) from tumor to capsule is defined as the shortest distance from the tumor boundary to the capsule or trachea.

Results: The multivariate logistic regression analysis indicated that age, gender, tumor maximum diameter, tumor mean diameter, and tumor volume were independently associated with CLNM. In the 692 cases without capsular invasion, the distance from the capsule was not correlated to the CLNM. The joint model, which included age, gender, tumor volume, and capsular invasion, were analyzed using the ROC curve. The cut-off point for the prediction of CLNM was defined as a value of 0.208 . The area under the ROC curve was 0.687 , the sensitivity was $65.4 \%$, and the specificity was $63.3 \%$.

Conclusions: Gender, age, maximum diameter, mean diameter, tumor volume, and capsular invasion were independently associated with the CLNM. When there was no capsular invasion, the distance between the tumor and capsule was not correlated to the CLNM, suggesting that considering whether the tumor is close to the capsule may not be necessary for low-risk PTMC.

Keywords: Papillary thyroid microcarcinoma (PTMC); distance; thyroid capsule; central lymph node metastasis; predictive factors

Submitted Mar 11, 2020. Accepted for publication Jun 04, 2020.

doi: 10.21037 /gs-20-478

View this article at: http://dx.doi.org/10.21037/gs-20-478 


\section{Introduction}

In recent years, the incidence of thyroid cancer has significantly increased worldwide, and most of which are papillary thyroid carcinomas (PTCs) (1-5). The main reason is the incidence of papillary thyroid microcarcinoma (PTMC, a papillary thyroid with a maximum tumor diameter of $\leq 10 \mathrm{~mm}$ ), which has rapidly increased $(6,7)$. Therefore, the treatment of PTMC has attracted the attention of researchers. At present, most scholars continue to insist that although PTMC has a good prognosis, most patients still required surgery. The 2015 American Thyroid Association (ATA) recommendations for PTMC requiring surgical treatment indicate that routine preventive dissection of lymph nodes is not recommended for T1 and T2 (8), which differs from the recommendations in the 2016 Chinese expert consensus for central lymph node dissection (9). Therefore, it remains controversial whether to perform prophylactic central lymph node dissection (PCND) in patients with PTMC. To date, no overwhelming evidence has proven whether PCND definitively improves PTMC patient prognosis (10-12). Some researchers have reported that the outcomes of thyroidectomy and thyroidectomy plus PCND are similar for experienced surgeons $(13,14)$. However, many studies have indicated that there is indeed an association between PCND and postoperative complications $(15,16)$. The PCND and postoperative complications of these published studies are summarized in Table 1 .

Though the PCND postoperative complications have been reduced in recent years, they still occur. Therefore, it is necessary to determine whether patients need PCND. The present study conducted a large sample retrospective study to explore the predictive factors of central lymph node metastasis (CLNM) in patients with PTMC in the Chinese population. There are similar articles $(21,27)$, but they are for PTC patients with tumor of less than $1.5 \mathrm{~cm}$ and tumor of $1-3 \mathrm{~cm}$ while this study was aimed at PTMC patients. And it is worth mentioning that the distance between tumor and capsule was taken as a parameter.

We present the following article in accordance with the STROBE reporting checklist (available at http://dx.doi. org/10.21037/gs-20-478).

\section{Methods}

\section{Study design and patients}

The study was conducted in accordance with the
Declaration of Helsinki and was approved by Medical Ethics Committee of Zhejiang Cancer Hospital (approval ID: IRB-2020-89). Informed consent was obtained in all cases for the study.

One thousand six hundred twenty-two PTMC cases in Zhejiang Cancer Hospital from January 2016 to October 2018 were enrolled in the present study. Then, the demographic and clinical information of these patients were obtained from the clinical medical records of our hospital. Inclusion criteria: (I) patients with tumor located on one side, with a maximum diameter of $\leq 10 \mathrm{~mm}$; (II) patients who underwent central lymph node dissection; (III) patients with a postoperative pathology confirmed as PTMC; (IV) patients who underwent operations performed by veteran surgeons; (V) patients who underwent an ultrasonography examination in Zhejiang Cancer hospital before undergoing surgery. Exclusion criteria: (I) patients who did not undergo a B-ultrasound examination in our hospital or had missing B-ultrasound images in our hospital; (II) patients who had lesions located on two sides; (III) patients with a lesion maximum diameter of $>10 \mathrm{~mm}$ under B-ultrasound; (IV) patients who previously received thyroid cancer surgery.

For patients with missing data: these patients were excluded or not included in the present study.

The study parameters included: age, gender, average tumor diameter (the three-dimensional data of the tumor was taken by ultrasonography), while the mean was calculated as the average diameter of the tumor. Tumor volume was calculated with the approximate volume using the sphere volume formula, $V=\pi / 6 \times$ average diameter ${ }^{3}$, regarding the tumor as the sphere. Capsular invasion and the distance from the capsule were taken on the ultrasonography. Research the correlation between distance and CLNM in patients without capsule invasion. This distance was the shortest distance from the tumor boundary to the capsule or trachea. We measured the distance between tumor and capsule on ultrasound image (double headed arrow (Figure 1). Age was categorized as follows: $\leq 45$ and $>45$ years old. The largest diameter of the tumor was classified as follows: $\leq 5$ and $>5 \mathrm{~mm}$. The average tumor diameter was classified as follows: $\leq 5$ and $>5 \mathrm{~mm}$. The tumor volume was classified as follows: $\leq 90$ and $>90 \mathrm{~mm}^{3}$. The distance from the capsule was classified as follows: $0-1$, $1-1.5,1.5-2.5$, and $2.5 \mathrm{~mm}$ or more.

\section{Statistical analysis}

The statistical analysis was performed using SPSS 23.0 
Table 1 The PCND and postoperative complications of the published studies

\begin{tabular}{|c|c|c|c|c|c|c|}
\hline Author & Year & Country & Study design & Case number & Surgical intervention & Complications \\
\hline $\operatorname{Lim}(18)$ & 2009 & Korea & Retrospective & 86 & $T$ + bilateral PCND & 49 \\
\hline So (19) & 2010 & Korea & Retrospective & 551 & $\pi$ + bilateral PCND & 192 \\
\hline Caliskan (20) & 2012 & Korea & Retrospective & 842 & TT/NTT + ipsilateral PCND & 179 \\
\hline Mao (22) & 2015 & China & Retrospective & 332 & $T T+$ ipsilateral or bilateral PCND & 81 \\
\hline Chang (23) & 2016 & Korea & Retrospective & 613 & $\begin{array}{l}\text { Hemithyroidectomy or TT + ipsilateral CLND or bilateral } \\
\text { CLND }\end{array}$ & 93 \\
\hline Yu (24) & 2017 & China & Retrospective & 917 & $\begin{array}{l}\text { Hemithyroidectomy plus prophylactic ipsilateral CLND or TT } \\
\text { plus a bilateral CLNM }\end{array}$ & 187 \\
\hline Luo (26) & 2019 & China & Retrospective & 1,031 & $\begin{array}{l}\text { Ipsilateral lobe and isthmus resection or } \pi T+\text { ipsilateral } \\
\text { CLND or bilateral CLND }\end{array}$ & 41 \\
\hline
\end{tabular}

TT, total thyroidectomy; PCND, prophylactic central lymph node dissection; NTT, not total thyroidectomy (less than total thyroidectomy); CLND, central lymph node dissection; CLNM, central lymph node metastasis.

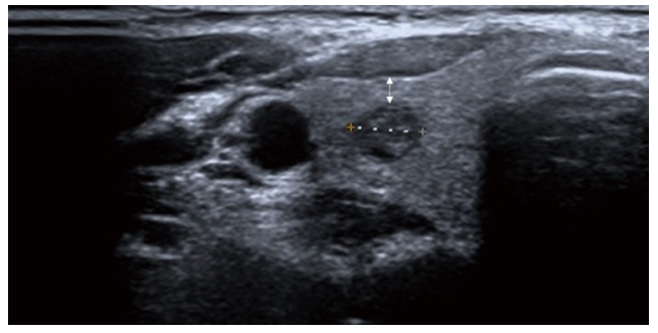

Figure 1 Measurement of distance between tumor to capsule (double headed arrow).

(IBM Corp., Armonk, NY, USA). All significance tests were two-sided. $\mathrm{P}<0.05$ was considered statistically significant. The CLNM was the dependent variable, with the negative converted to 0 and the positive converted to 1 . The univariate analysis with the $\chi^{2}$-test was used to analyze the statistical correlation between the factors and CLNM. The multivariate logistic regression analysis was performed to find the multivariate correlation of the CLNM. The results were presented in odds ratio (OR) with a $95 \%$ confidence interval $(\mathrm{CI})$ and a $\mathrm{P}$ value. The nomogram for predicting the CLNM was developed based on gender, age, capsular invasion, and tumor volume. The predictive value of the model was measured using the area under the receiver operating characteristic (ROC) curve. Rstudio was used to construct a nomogram to predict the CLNM and calibration plot based on gender, age, tumor volume, and capsular invasion.

\section{Results}

\section{Patients characteristics}

From January 2016 to October 2018, 1,622 patients with PTMC, who underwent surgical treatment at Zhejiang Cancer Hospital, were retrospectively assessed (Table 2). The mean age of these patients was $44.5 \pm 10.8$ years old, which ranged between $17-80$ years old. The mean diameter of the tumor was $5.1 \pm 1.6 \mathrm{~mm}$, the mean maximum diameter of the tumor was $5.8 \pm 3.2 \mathrm{~mm}$, the mean tumor volume was $92.5 \pm 83.7 \mathrm{~mm}^{3}$, and 930 cases had a capsular invasion. For the remaining 692 cases with noninvasive capsules, the mean distance from the capsule was $2.0 \pm 1.2 \mathrm{~mm}$, which ranged from 0.5 to $9.7 \mathrm{~mm}$.

\section{Univariate and multivariate analysis}

For the univariate multivariable analysis, age $\leq 45$ years old $(\mathrm{P}<0.001)$, male gender $(\mathrm{P}<0.001)$, a larger mean diameter ( $\leq 5 v s .>5 \mathrm{~mm}, \mathrm{P}<0.001)$, a larger maximum diameter $(\leq 5$ vs. $>5 \mathrm{~mm}, \mathrm{P}<0.001)$, a larger tumor volume ( $\leq 90 v s .>90 \mathrm{~mm}^{3}$, 
Table 2 Clinicopathological characteristics of the study patients

\begin{tabular}{|c|c|}
\hline Clinical pathological factors & Number of cases (\%) \\
\hline \multicolumn{2}{|l|}{ Age (years) } \\
\hline$\leq 45$ & $846(52.2)$ \\
\hline$>45$ & $776(47.8)$ \\
\hline \multicolumn{2}{|l|}{ Gender } \\
\hline Male & $362(22.3)$ \\
\hline Female & $1,260(77.7)$ \\
\hline \multicolumn{2}{|l|}{ Mean diameter (mm) } \\
\hline$\leq 5$ & $786(48.5)$ \\
\hline$>5$ & $836(51.5)$ \\
\hline \multicolumn{2}{|l|}{ Maximum diameter (mm) } \\
\hline$\leq 5$ & $568(35.0)$ \\
\hline$>5$ & $1,054(65.0)$ \\
\hline \multicolumn{2}{|l|}{ Tumor volume $\left(\mathrm{mm}^{3}\right)$} \\
\hline$\leq 90$ & $944(58.2)$ \\
\hline$>90$ & $678(41.8)$ \\
\hline \multicolumn{2}{|l|}{ Capsule invasion } \\
\hline No invasion & $692(42.7)$ \\
\hline Invasion & $930(57.3)$ \\
\hline \multicolumn{2}{|l|}{ Distance from the capsule } \\
\hline$\leq 1 \mathrm{~mm}$ & $128(18.5)$ \\
\hline $1.0-1.5 \mathrm{~mm}$ & $161(23.3)$ \\
\hline $1.5-2.5 \mathrm{~mm}$ & $228(32.9)$ \\
\hline$>2.5 \mathrm{~mm}$ & $175(25.3)$ \\
\hline
\end{tabular}

$\mathrm{P}<0.001)$, and capsular invasion $(\mathrm{P}=0.011)$ were significantly associated with the CLNM (Table 3). For the case of patients without capsular invasion, the tumor distance from the capsule was not statistically significant $(\mathrm{P}>0.05)$.

For all cases, each factor was included in a two-class logistic regression multivariate analysis (Table 4). The dependent variable was CLNM, and the independent variables were age, gender, average tumor diameter, tumor volume, and capsular invasion. The risk of CLNM for an average diameter of $>5 \mathrm{~mm}$ is 1.967 times of that for a diameter of $\leq 5 \mathrm{~mm}$. The risk of CLNM resulted in tumor $>5 \mathrm{~mm}$ is 2.060 times of tumor $\leq 5 \mathrm{~mm}$. A tumor volume of $>90 \mathrm{~mm}^{3}$ was 1.90 times of tumor volumes of $\leq 90 \mathrm{~mm}^{3}$. The risk of CLNM with capsular invasion was 1.391 times of that of noninvasive capsules. The tumor size and distances from the capsule on the ultrasonography are presented in Figure 2. The distance was not statistically significant in patients without capsular invasion.

\section{Nomogram creation}

The nomogram was created with the predictive variables from the statistical analysis. The $\operatorname{Logit}(\mathrm{P})=-0.461+$ $0.903 \times$ gender (when the gender was male) -0.036 $\times$ age $+0.159 \times($ if with capsular invasion $)+0.004 \times$ tumor volume $\left(\mathrm{mm}^{3}\right)$ was determined, and the predicted probability $\left(P=\frac{e^{\operatorname{Logit}(P)}}{1+e^{\operatorname{Logit}(P)}}\right)$ was obtained. Based on this, the ROC curve was drawn (Figure 3). The area under the ROC curve was 0.687 . According to the corresponding sensitivity and specificity of each point, calculate the point with the maximum value of (sensitivity + specificity-1) as cut off value. A cut-off point for the Prediction of the CLNM was defined as 0.208 . The sensitivity was $65.4 \%$, while the specificity was $63.3 \%$. On the basis of multi factor regression analysis, multiple prediction indexes are integrated to get the nomogram (Figure 4). And we verified the prediction ability of the prediction model by internal verification (Figure 5). From these, it could be concluded that the combination of gender, age, capsular invasion, and tumor volume has a certain predictive value for CLNM.

\section{Discussion}

Controversy still exists on whether PTMC surgery requires PCND. Therefore, searching for predictors of CLNM in PTMC has great significance for the choice of PCND. At present, there is a consensus on the predictability of gender, age, the maximum diameter of the tumor, and capsular invasion for the CLNM of PTMC (26,28-33). The result of the present study was consistent with this. Additionally, the present study included the average diameter, tumor volume, and distance from the capsule.

Tumor size is presently considered a predictor of CLNM. Most studies have taken the largest diameter of the tumor as the tumor size. Some studies have suggested that tumors $>8.5 \mathrm{~mm}$ are more aggressive and have a worse prognosis (34). Some studies have suggested that the critical value of PTMC invasiveness could be considered as $7 \mathrm{~mm}$ (35). There is no definitive conclusion at present. Furthermore, there are few studies on the predictive significance of approximate tumor volume for CLNM. The 
Table 3 Univariate analysis of the clinical pathological factors for central lymph node metastasis

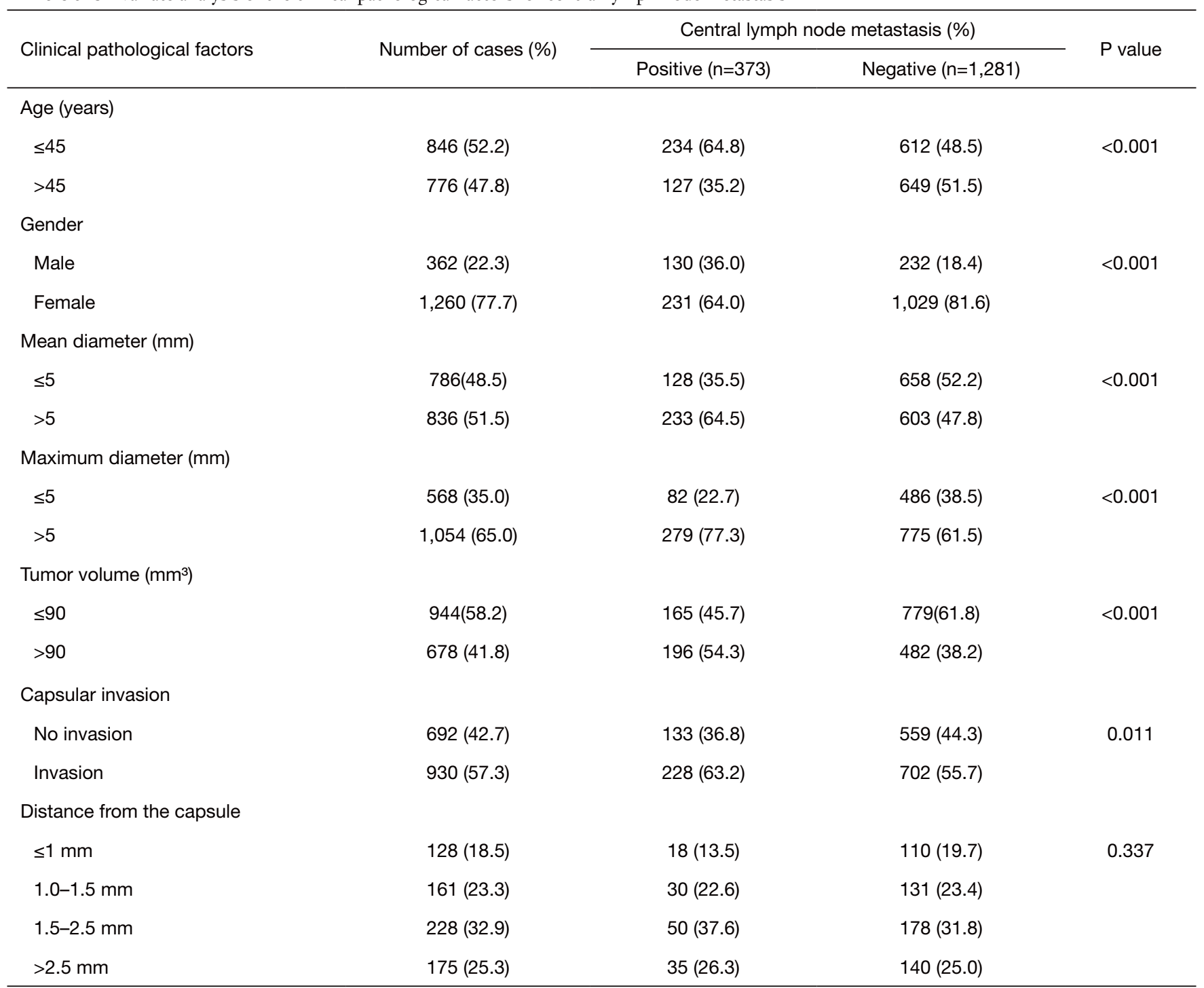

Table 4 Multivariate analysis of the clinical pathological factors for central lymph node metastasis

\begin{tabular}{lccc}
\hline \multirow{2}{*}{ Variable } & \multicolumn{2}{c}{ Central lymph node metastasis risk } & \multicolumn{1}{c}{$\mathrm{P} \% \mathrm{Cl}$} \\
\hline Age & OR & $0.400-0.656$ & $<.001$ \\
Gender & 0.512 & $1.959-3.309$ & $<0.001$ \\
Mean diameter & 2.546 & $1.539-2.513$ & $<0.001$ \\
Maximum diameter & 1.967 & $1.568-2.708$ & $<0.001$ \\
Tumor volume & 2.060 & $1.498-2.414$ & $<0.001$ \\
Capsular invasion & 1.902 & $1.068-1.781$ & 0.009 \\
Distance from the capsule & 1.391 & $0.911-1.324$ & 0.325 \\
\hline
\end{tabular}

OR, odds ratio; $\mathrm{Cl}$, confidence interval. 


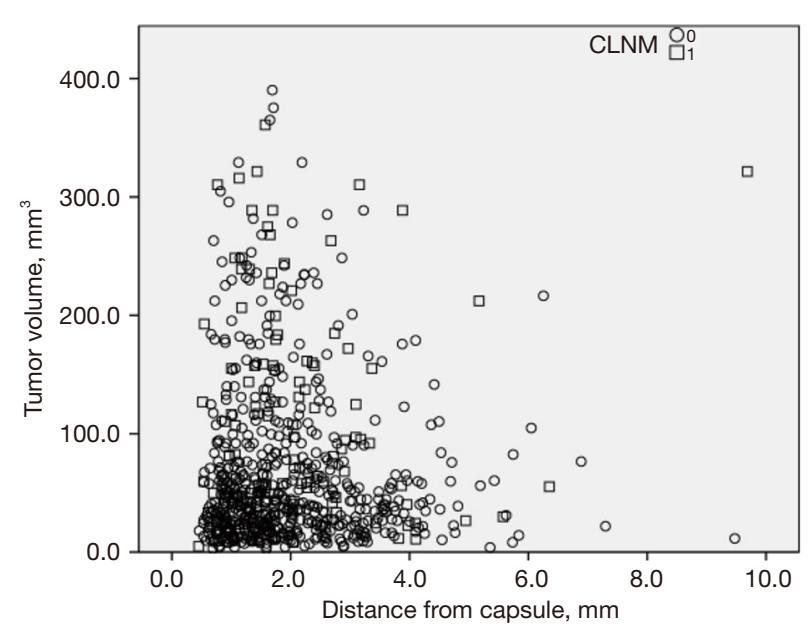

Figure 2 Tumor size and the distance from the capsule on the ultrasonography images. The Pearson correlation was -0.15 . Combined with Figure 1, it can be concluded that the correlation between the two is not strong.

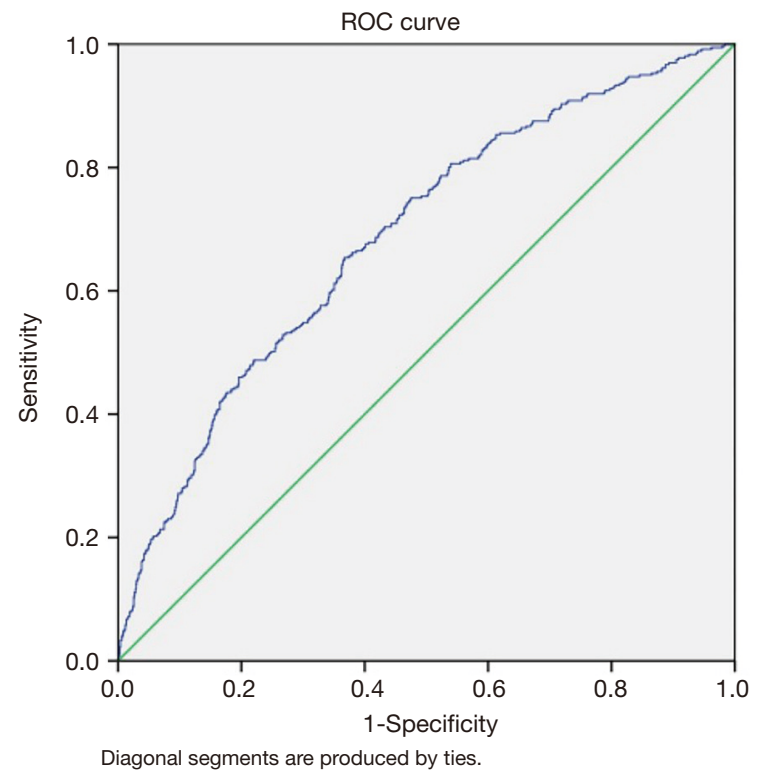

Figure 3 The area under the ROC curve was 0.687. The cut-off points for the Prediction of the CLNM were defined as 0.208 . The sensitivity was $65.4 \%$, while the specificity was $63.3 \%$. ROC, receiver operating characteristic; CLNM, central lymph node metastasis.

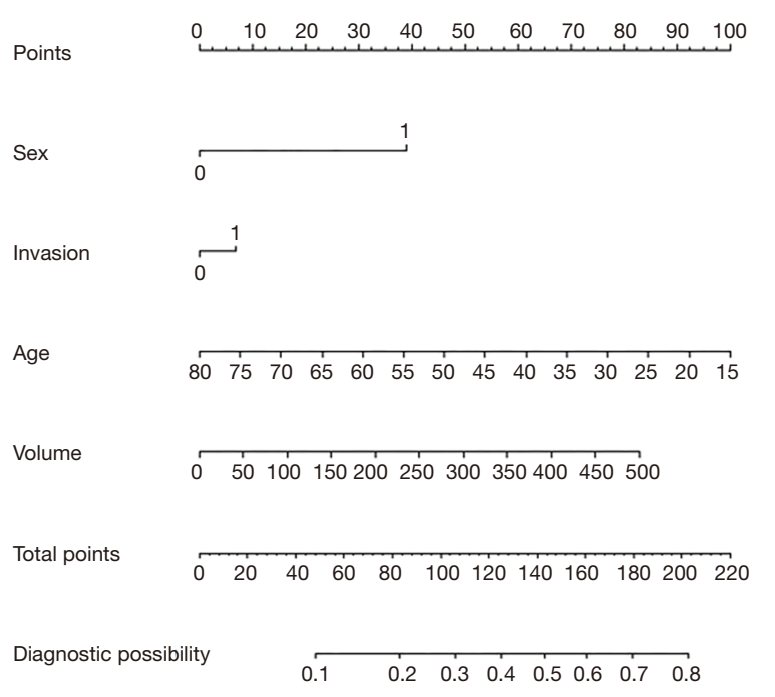

Figure 4 The nomogram to estimate the risk of CLNM based on gender, age, tumor volume, and capsular invasion. CLNM, central lymph node metastasis.

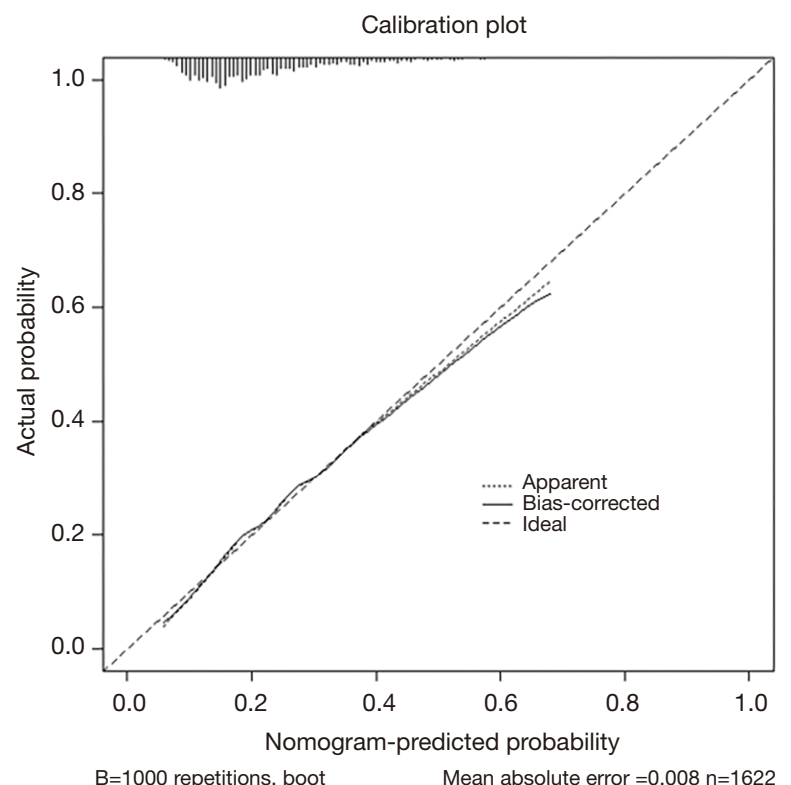

Figure 5 The calibration plot for predicting the performance of the nomogram in estimating the risk of CLNM. When the nomogram-predicted probability was lower than 0.4 , the ideal value was close to the actual value. When the nomogram-predicted probability was higher than 0.4 , the ideal value is higher. CLNM, central lymph node metastasis. 
current study was based on the predictive tumor size for CLNM, and the volume of the tumor was determined using the mean tumor diameter measured on ultrasonography. Considering the tumor as the sphere to obtain the approximate volume, although there was a certain amount of error, this can still be used as a supplementary reference index for the maximum diameter, in order to reduce the possible error. A study revealed that the tumor volume on ultrasonography is a predictor of central neck metastasis of PTC (36). Some studies used the largest tumor diameter and tumor volume to evaluate the size of PTMC tumors. These studies showed that the tumor volume is more sensitive than the maximum diameter of the tumor during the follow-up time for low-risk PTC and that the tumor size change would be more timely. Some scholars have considered that in PTC, the volume is more accurate than the largest diameter to evaluate the tumor size $(37,38)$. Although our study did not demonstrate that the tumor volume is more sensitive than the maximum tumor diameter in the prediction of CLNM (39), the data analysis indicated that tumor volume is a predictor of CLNM. The combination of volume and maximum diameter can more fully and accurately describe the tumor size, and guide for determining whether to perform PCND. In combining these two, the tumor size can be more fully and accurately expressed.

The nomogram revealed that it has predictive performance. The predicted probability can be calculated by combining age, gender, capsular invasion, and tumor volume. Although the sensitivity and specificity were not high, this can be a reference factor for PCND.

The relationship between the thyroid capsule and CLNM has been the focus of many scholars. Most scholars have studied the predictability of capsular invasion for CLNM, and have a common understanding that capsular invasion is a predictor of CLNM (26,29-33). Most scholars have the common understanding that tumor capsular invasion can predict the CLNM of thyroid cancer. Furthermore, some scholars even consider that determining whether the tumor in contact with the capsule more than $25 \%$ is predictive of CLNM of PTMC $(40,41)$. Also, some scholars have also studied extracapsular extension, which refers to the extrathyroid extension, or even when the tumor destroys the thyroid capsule, it is considered to be an independent influencing factor of CLNM $(25,42,43)$. However, there are few studies on the correlation of the distance from the capsule for CLNM. Some scholars have selected more than $1 \mathrm{~cm}$ of PTC in their investigations and selected the shortest distance from the capsule on ultrasonography. It has been considered that the distance has a specific significance for predicting the CLNM of PTC. Furthermore, it has been considered that both univariate multivariable analysis and chi-square $\left(\chi^{2}\right)$ showed a distance from the capsule $<1.9 \mathrm{~mm}$ is an important index for the non-metastasis of CLNM $(\mathrm{P}<0.05)$ (27). Some scholars had similar conclusions, using spearman correlation analysis, the shorter distance from tumor to capsule, the greater risk in developing of CLNM(spearman correlation coefficient $=-0.22, \mathrm{P}<0.0001)(44)$. It is worth mentioning that the former included PTC patients with tumor of $1-3 \mathrm{~cm}$, while the latter included PTC patients with tumor of 0.1-5.6 $\mathrm{cm}$, and the distance $\geq 0$. These are the differences from our study. And when choosing the low-risk PTMC which can be followed up instead of surgery, the PTMC whose tumor is close to the capsule or trachea is excluded. These make us suspect that distance may be a risk factor for CLNM in PTMC patients. However the present study revealed that the distance between the tumor and capsule has no significant predictive value for the CLNM of PTMC. Therefore, as far as the result of the present study is concerned, the distance between the tumor and capsule is not a guiding factor for determining whether PTMC needs PCND.

Scholars have proposed the theory of low-risk PTMC, and have considered that it can be followed up for an extended period, instead of immediately operating. Furthermore, some scholars have proposed a risk stratification for patients who are considered for active monitoring. Some factors include the location of the tumor and an ideal follow-up population. For example, it is surrounded by $\geq 2 \mathrm{~mm}$ of normal thyroid parenchyma. The subcapsular position is appropriate; the position is not adjacent to the recurrent laryngeal nerve, without evidence of extrathyroidal extension. Likewise, it is not inappropriate; subcapsular locations are adjacent to the recurrent laryngeal nerve. These result in evidence of extrathyroid extension or clinical evidence of invasion to the recurrent laryngeal nerve or trachea (45). Low-risk PTMC is also mentioned in the 2016 Chinese expert consensus and guidelines for the diagnosis and treatment of PTC, and it was considered that low-risk PTMC could be followed should satisfy the condition that the tumor is not close to the capsule (9). However, indeed, no study has determined whether the distance between the capsule and tumor within the thyroid gland is a high-risk factor. The result of the present study suggests that when the tumor of the PTMC does not invade the capsule, the closest distance from the 
tumor to the capsule has no association with the CLNM. Therefore, when screening for low-risk PTMC, it should be considered whether the tumor invades the capsule. If there is no invasion, it would not be meaningful to determine whether the tumor is close to the capsule. China has not yet conducted a long-term follow-up of low-risk PTMC. According to the results of the present study, the follow-up range for low-risk PTMC can be appropriately expanded. This means that more patients can be followed-up with instead of undergoing surgery.

It is worth noting some of the limitations of the present study. First, it can be observed in Figure 2 that although there were 692 cases of PTMC with tumors within the gland selected in the present study, most of which were small in size and had a short distance from the capsule. Hence, it may not be possible to explore the relevance of distance to CLNM in more detail. Second, although 692 cases of PTMC without capsule invasion were selected for the present study, there was a certain amount of error in the measurement on the ultrasonography image. It can be observed that the closest distance in the present study was $0.5 \mathrm{~mm}$, and there was a possibility that the closer distance $(<0.5 \mathrm{~mm})$ was classified as a capsular invasion. However, ultrasonography is an essential method for PTMC, and it is also the most effective and accurate method for measuring the distance between the tumor and capsule. Another limitation is that the present study verified the correlation between tumor volume and CLNM but did not compare tumor volume to the maximum diameter, which is more predictive of CLNM.

In conclusion, the present retrospective study proved that gender, age, maximum diameter, mean diameter, tumor volume, and capsular invasion are predictors of CLNM. When there is no capsular invasion, the distance between the tumor and capsule did not correlate to CLNM. Lowrisk PTMC does not need to consider whether the tumor is close to the capsule.

\section{Acknowledgments}

We thank all the patients who participated in this study.

Funding: This work was supported by the Zhejiang Provincial Natural Science Foundation of China (LY20H160007).

\section{Footnote}

Reporting Checklist: The authors have completed the
STROBE reporting checklist. Available at http://dx.doi. org/10.21037/gs-20-478

Data Sharing Statement: Available at http://dx.doi. org/10.21037/gs-20-478

Conflicts of Interest: All authors have completed the ICMJE uniform disclosure form (available at http://dx.doi. org/10.21037/gs-20-478). The authors have no conflicts of interest to declare.

Ethical Statement: The authors are accountable for all aspects of the work in ensuring that questions related to the accuracy or integrity of any part of the work are appropriately investigated and resolved. The study was conducted in accordance with the Declaration of Helsinki and was approved by Medical Ethics Committee of Zhejiang Cancer Hospital (approval ID: IRB-2020-89). Informed consent was obtained in all cases for the study.

Open Access Statement: This is an Open Access article distributed in accordance with the Creative Commons Attribution-NonCommercial-NoDerivs 4.0 International License (CC BY-NC-ND 4.0), which permits the noncommercial replication and distribution of the article with the strict proviso that no changes or edits are made and the original work is properly cited (including links to both the formal publication through the relevant DOI and the license). See: https://creativecommons.org/licenses/by-nc-nd/4.0/.

\section{References}

1. Davies L, Welch HG. Current Thyroid Cancer Trends in the United States. Jama Otolaryngology-Head \& Neck Surgery 2014;140:317.

2. Ullmann TM, Gray KD, Moore MD, et al. Current controversies and future directions in the diagnosis and management of differentiated thyroid cancers. Gland Surg 2018;7:473-86.

3. Wiltshire JJ, Drake TM, Uttley L, et al. Systematic Review of Trends in the Incidence Rates of Thyroid Cancer. Thyroid 2016;26:1541-52.

4. Shaha AR, Tuttle RM. Thyroid cancer staging and genomics. Ann Transl Med 2019;7:S49.

5. Morris LG, Sikora AG, Tosteson TD, et al. The increasing incidence of thyroid cancer: the influence of access to care. Thyroid 2013;23:885-91.

6. Du L, Wang Y, Sun X, et al. Thyroid cancer: trends in 
incidence, mortality and clinical-pathological patterns in Zhejiang Province, Southeast China. BMC Cancer 2018;18:291.

7. Jung CK, Little MP, Lubin JH, et al. The increase in thyroid cancer incidence during the last four decades is accompanied by a high frequency of BRAF mutations and a sharp increase in RAS mutations. J Clin Endocrinol Metab 2014;99:E276-85.

8. Haugen BR, Alexander EK, Bible KC, et al. 2015 American Thyroid Association Management Guidelines for Adult Patients with Thyroid Nodules and Differentiated Thyroid Cancer: The American Thyroid Association Guidelines Task Force on Thyroid Nodules and Differentiated Thyroid Cancer. Thyroid 2016;26:1-133.

9. Chai L, Han D, Li J, et al. The construction and analysis of gene co-expression network of differentially expressed genes identifies potential biomarkers in thyroid cancer. Transl Cancer Res 2018;7:1235-43.

10. Qu N, Zhang L, Ji QH, et al. Number of tumor foci predicts prognosis in papillary thyroid cancer. BMC Cancer 2014;14:914.

11. Wada N, Duh QY, Sugino K, et al. Lymph node metastasis from 259 papillary thyroid microcarcinomas: frequency, pattern of occurrence and recurrence, and optimal strategy for neck dissection. Ann Surg 2003;237:399-407.

12. Noguchi S, Yamashita H, Uchino S, et al. Papillary microcarcinoma. World J Surg 2008;32:747-53.

13. Fritze D, Doherty GM. Surgical management of cervical lymph nodes in differentiated thyroid cancer. Otolaryngol Clin North Am 2010;43:285-300, viii.

14. Carty SE, Cooper DS, Doherty GM, et al. Consensus statement on the terminology and classification of central neck dissection for thyroid cancer. Thyroid 2009;19:1153-8.

15. Roh JL, Park JY, Park CI. Total thyroidectomy plus neck dissection in differentiated papillary thyroid carcinoma patients: pattern of nodal metastasis, morbidity, recurrence, and postoperative levels of serum parathyroid hormone. Ann Surg 2007;245:604-10.

16. Cavicchi O, Piccin O, Caliceti U, et al. Transient hypoparathyroidism following thyroidectomy: a prospective study and multivariate analysis of 604 consecutive patients. Otolaryngol Head Neck Surg 2007;137:654-8.

17. Lee SH, Lee SS, Jin SM, et al. Predictive factors for central compartment lymph node metastasis in thyroid papillary microcarcinoma. Laryngoscope 2008;118:659-62.
18. Lim YC, Choi EC, Yoon YH, et al. Central lymph node metastases in unilateral papillary thyroid microcarcinoma. Br J Surg 2009;96:253-7.

19. So YK, Son YI, Hong SD, et al. Subclinical lymph node metastasis in papillary thyroid microcarcinoma: a study of 551 resections. Surgery 2010;148:526-31.

20. Caliskan M, Park JH, Jeong JS, et al. Role of prophylactic ipsilateral central compartment lymph node dissection in papillary thyroid microcarcinoma. Endocr J 2012;59:305-11.

21. Park JP, Roh JL, Lee JH, et al. Risk factors for central neck lymph node metastasis of clinically noninvasive, nodenegative papillary thyroid microcarcinoma. Am J Surg 2014;208:412-8.

22. Mao LN, Wang P, Li ZY, et al. Risk factor analysis for central nodal metastasis in papillary thyroid carcinoma. Oncol Lett 2015;9:103-7.

23. Chang YW, Kim HS, Kim HY, et al. Should central lymph node dissection be considered for all papillary thyroid microcarcinoma? Asian J Surg 2016;39:197-201.

24. Yu X, Song X, Sun W, et al. Independent Risk Factors Predicting Central Lymph Node Metastasis in Papillary Thyroid Microcarcinoma. Horm Metab Res 2017;49:201-7.

25. Gui CY, Qiu SL, Peng ZH, et al. Clinical and pathologic predictors of central lymph node metastasis in papillary thyroid microcarcinoma: a retrospective cohort study. J Endocrinol Invest 2018;41:403-9.

26. Luo Y, Zhao Y, Chen K, et al. Clinical analysis of cervical lymph node metastasis risk factors in patients with papillary thyroid microcarcinoma. J Endocrinol Invest 2019;42:227-36.

27. Seong CY, Chai YJ, Lee SM, et al. significance of distance between tumor and thyroid capsule as an indicator for central lymph node metastasis in clinically node negative papillary thyroid carcinoma patients. PLoS One 2018;13:e0200166.

28. Wu X, Li B, Zheng C, et al. Risk factors for central lymph node metastases in patients with papillary thyroid microcarcinoma. Endocr Pract 2018;24:1057-62.

29. Zhang Q, Wang Z, Meng X, et al. Predictors for central lymph node metastases in CN0 papillary thyroid microcarcinoma (mPTC): A retrospective analysis of 1304 cases. Asian J Surg 2019;42:571-6.

30. Vasileiadis I, Karakostas E, Charitoudis G, et al. Papillary thyroid microcarcinoma: clinicopathological characteristics and implications for treatment in 276 patients. Eur J Clin Invest 2012;42:657-64. 
31. Sun W, Lan X, Zhang H, et al. Risk Factors for Central Lymph Node Metastasis in CN0 Papillary Thyroid Carcinoma: A Systematic Review and Meta-Analysis. PLoS One 2015;10:e139021.

32. Xu D, Lv X, Wang S, et al. Risk factors for predicting central lymph node metastasis in papillary thyroid microcarcinoma. Int J Clin Exp Pathol 2014;7:6199-205.

33. Huang XP, Ye TT, Zhang L, et al. Sonographic features of papillary thyroid microcarcinoma predicting highvolume central neck lymph node metastasis. Surg Oncol 2018;27:172-6.

34. Gong Y, Li G, Lei J, et al. A favorable tumor size to define papillary thyroid microcarcinoma: an analysis of 1176 consecutive cases. Cancer Manag Res 2018;10:899-906.

35. Lee KJ, Cho YJ, Kim SJ, et al. Analysis of the clinicopathologic features of papillary thyroid microcarcinoma based on 7-mm tumor size. World J Surg 2011;35:318-23.

36. Park KN, Kang KY, Hong HS, et al. Predictive Value of Estimated Tumor Volume Measured by Ultrasonography for Occult Central Lymph Node Metastasis in Papillary Thyroid Carcinoma. Ultrasound Med Biol 2015;41:2849-54.

37. Kwon H, Oh HS, Kim M, et al. Active Surveillance for Patients With Papillary Thyroid Microcarcinoma: A Single Center's Experience in Korea. J Clin Endocrinol Metab 2017;102:1917-25.

38. Tuttle RM, Fagin JA, Minkowitz G, et al. Natural History and Tumor Volume Kinetics of Papillary Thyroid Cancers During Active Surveillance. JAMA Otolaryngol Head Neck Surg 2017;143:1015-20.

Cite this article as: Zhu M, Zheng W, Xiang Y, Gu J, Wang $\mathrm{K}$, Shang J. The relationship between central lymph node metastasis and the distance from tumor to thyroid capsule in papillary thyroid microcarcinoma without capsule invasion. Gland Surg 2020;9(3):727-736. doi: 10.21037/gs-20-478
39. Pennington TE, Thwin M, Sywak M, et al. Sonographic Volumetric Assessment Is a More Accurate Measure Than Maximum Diameter Alone in Papillary Thyroid Cancer. J Endocr Soc 2018;2:1284-92.

40. Jin WX, Ye DR, Sun YH, et al. Prediction of central lymph node metastasis in papillary thyroid microcarcinoma according to clinicopathologic factors and thyroid nodule sonographic features: a case-control study. Cancer Manag Res 2018;10:3237-43.

41. Cai YF, Wang QX, Ni CJ, et al. A scoring system is an effective tool for predicting central lymph node metastasis in papillary thyroid microcarcinoma: a case-control study. World J Surg Oncol 2016;14:45.

42. Siddiqui S, White MG, Antic T, et al. Clinical and Pathologic Predictors of Lymph Node Metastasis and Recurrence in Papillary Thyroid Microcarcinoma. Thyroid 2016;26:807-15.

43. Liu LS, Liang J, Li JH, et al. The incidence and risk factors for central lymph node metastasis in cN0 papillary thyroid microcarcinoma: a meta-analysis. Eur Arch Otorhinolaryngol 2017;274:1327-38.

44. Wang QC, Cheng W, Wen X, et al. Shorter distance between the nodule and capsule has greater risk of cervical lymph node metastasis in papillary thyroid carcinoma. Asian Pac J Cancer Prev 2014;15:855-60.

45. Brito JP, Ito Y, Miyauchi A, Tuttle RM. A Clinical Framework to Facilitate Risk Stratification When Considering an Active Surveillance Alternative to Immediate Biopsy and Surgery in Papillary Microcarcinoma. Thyroid 2016;26:144-9. 